\title{
TROP2: a novel prognostic marker in squamous cell carcinoma of the oral cavity
}

\author{
Dominic Fong ${ }^{1,2}$, Gilbert Spizzo ${ }^{1,2}$, Johanna M Gostner ${ }^{2}$, Guenther Gastl ${ }^{1,2}$, Patrizia Moser ${ }^{3}$, \\ Clemens Krammel ${ }^{2}$, Stefan Gerhard ${ }^{4}$, Michael Rasse ${ }^{4}$ and Klaus Laimer ${ }^{2,4}$ \\ ${ }^{1}$ Department of Hematology and Oncology, Innsbruck Medical University, Innsbruck, Austria; ${ }^{2}$ Tyrolean \\ Cancer Research Institute, Innsbruck, Austria; ${ }^{3}$ Department of Pathology, Innsbruck Medical University, \\ Innsbruck, Austria and ${ }^{4}$ Department of Maxillofacial Surgery, Innsbruck Medical University, Innsbruck, \\ Austria
}

\begin{abstract}
Squamous cell carcinoma is by far the most common type of cancer of the oral cavity, representing more than $90 \%$ of all oral cancers. Despite refinement of surgical techniques and adjuvant therapies, the prognosis for patients with oral squamous cell carcinoma remains poor. Identification of prognostic factors related to tumor biology might improve this assessment. Recently, the human trophoblast cell-surface antigen TROP2 was found to be highly expressed in colorectal cancer, correlating with aggressiveness and poor prognosis. Thus, the aim of this study was to investigate TROP2 expression and its prognostic impact in oral squamous cell carcinoma patients. TROP2 expression was examined by immunohistochemistry in a series of 90 patients on a tissue microarray of paraffin-embedded specimens. Survival was calculated using Kaplan-Meier estimates. Parameters found to be of prognostic significance in univariate analysis were verified in a multivariate Cox regression model. TROP2 overexpression was observed in $52(58 \%)$ of the tumor samples. Kaplan-Meier curves showed that TROP2 overexpression was significantly associated with decreased overall survival $(\boldsymbol{P}<0.01)$. Overall survival gradually worsened with increasing TROP2 scores. By univariate analyses, no correlation with conventional clinicopathological features was found. Multivariate Cox regression analysis revealed TROP2 overexpression to be an independent factor predictive of poor disease outcome $(P<0.01)$. These results demonstrate that TROP2 overexpression is an independent prognostic marker in patients with oral squamous cell carcinoma. TROP2 overexpression was detectable in $58 \%$ of the tumor samples, indicating it to be a potential novel therapeutic target in squamous cell carcinoma of the oral cavity.
\end{abstract}

Modern Pathology (2008) 21, 186-191; doi:10.1038/modpathol.3801001; published online 14 December 2007

Keywords: TROP2; OSCC; EpCAM; GA733; TACSTD; oral squamous cell carcinoma

Despite numerous advances in diagnosis and treatment of oral squamous cell carcinoma over the last decades, mortality rates have remained unchanged, with a 5-year survival rate of about $40-50 \%$. Currently, treatment decisions are based on established clinicopathological parameters like the TNM system. However, tumor progression seems to be a multifactorial and multistep process, resulting in a heterogeneous group of cancers. The better understanding of the molecular basis of tumor characteristics plays an important role in cancer drug development. Among the most exciting and promising benefits are molecular targeted cancer therapies. Therefore, there is a need for the search

Correspondence: Dr D Fong, MD, Department of Hematology and Oncology, Innsbruck Medical University, Anichstrasse 35, Innsbruck A-6020, Austria.

E-mail: dominic.fong@i-med.ac.at

Received 26 July 2007; revised 27 September 2007; accepted 01 October 2007; published online 14 December 2007 of new factors with therapeutic and prognostic relevance in order to individualize the management of patients with oral squamous cell carcinoma.

The human trophoblast cell-surface antigen TROP2 (also termed GA733-1, M1S1, EGP-1) is encoded by the TACSTD2 gene, which has been mapped to the human chromosome 1p32. ${ }^{1}$ The TACSTD gene family encodes at least two different integral membrane glycoproteins, termed TACSTD2 (TROP2) and TACSTD1 (EpCAM). The TACSTD2 gene is intronless, formed by exon shuffling and the retroposition of the TACSTD1 gene via an mRNA intermediate. ${ }^{2,3}$ It encodes a protein with several structural features in common with the transmembrane glycoprotein TACSTD1, such as an EGF-like repeat as well as a sequence homologous to the IL-2 receptor. ${ }^{3,4}$

TROP2, originally identified on human trophoblast and choriocarcinoma cell lines, was subsequently shown to be highly expressed by the majority of human carcinomas but rarely by normal 
tissues. ${ }^{5-7}$ The cytoplasmic tail contains phosphorylation sites functioning as a signal transducer. ${ }^{8,9}$ The function of TROP2 is not well understood, although it has previously been suggested to play a role in regulating the growth of carcinoma cells as well as in fetal lung formation. ${ }^{10-12}$ These findings suggest a potential role of TROP2 in tumor development. As a consequence of its wide distribution on epithelial tumors, TROP2 seems to be an attractive structure as a possible immunotherapeutic target. We therefore investigated TROP2 expression in oral squamous cell carcinoma and its correlation with clinicopathologic features.

\section{Materials and methods}

The study was conducted according to the regulations of the local ethics committee and Austrian Law. In this retrospective study, all patients who underwent surgery at the Department of Maxillofacial Surgery, Innsbruck Medical University, between 1980 and 1997 and for whom tissue specimens from the local pathology repository and clinical follow-up data were available were included. All tumors were reclassified on hematoxylin- and eosin-stained slides, and histological type and tumor grade were reassessed by a pathologist (PM) using standard diagnostic criteria. Clinical data were obtained by reviewing the charts and contacting the physicians-in-charge. Tumors were histologically classified according to the WHO classification and staged according to the International Union Against Cancer tumor-nodemetastasis classification (UICC).

\section{Tissue Microarray}

For tissue microarray construction, we used hematoxylin- and eosin-stained slides from each tumor block to select a morphologically representative tumor area. Tissue cylinders with a diameter of $2 \mathrm{~mm}$ were punched from the marked tumor area of each block (donor block) and brought into a recipient paraffin block (recipient block) using a precision instrument (Manual Tissue Arrayer, MTA-1, Beecher Instruments, Wisconsin, USA). Three different tissue microarrays were constructed, each containing about 30 punches of oral squamous cell carcinoma in a specific arraying pattern. Sections from these blocks were cut with a microtome and mounted on tissue microarray-specific adhesive-coated glass slides, which were used for the immunohistochemical analysis.

\section{Immunohistochemistry}

The expression of TROP2 was determined by immunohistochemistry using the purified goat polyclonal antibody against the recombinant human
TROP2 extracellular domain at a dilution of 1:50 (AF650, R\&D Systems Inc., Minneapolis, MN, USA). Briefly, 5- $\mu \mathrm{m}$ sections were cut from recipient paraffin blocks, mounted on tissue microarrayspecific adhesive-coated glass slides (Instrumedics, Missouri, USA), deparaffinized and rehydrated. Endogenous peroxidase was blocked with methanol containing $30 \%$ hydrogen peroxide for $20 \mathrm{~min}$. Pretreatment consisted of a $20 \mathrm{~min}$ incubation period in a pronase solution. After several washes, the sections were incubated with a biotinylated anti-goat antibody for $30 \mathrm{~min}$ (1:100; Ab6740, Abcam, Cambridge, England). Labeling for TROP2 was completed using the ABC reagent (Vectastain Elite ABC Kit Standard PK-6100, Vienna, Austria) for $30 \mathrm{~min}$ at room temperature. Visualization of immunolabeled structures was achieved by incubating the sections in diaminobenzidine $/ \mathrm{H}_{2} \mathrm{O}_{2}$ solution containing 30\% hydrogen peroxide until coloration was visible (usually 3-4 min). Finally, the slides were counterstained with Mayer's hemalaun solution, followed by clearing and mounting. Positive and negative controls were included in each run. Representative micrographs of tumors with predominant membranous staining of TROP2 are shown in Figure 1.

\section{Evaluation of Slides}

TROP2 overexpression was evaluated by two independent observers (PM and DF) using light microscope in a blinded fashion. Discordant cases were re-evaluated on a double-headed microscope to achieve a consensus. Antigen expression was defined as the presence of specific staining on the surface membrane of tumor cells. TROP2 overexpression was evaluated for each tissue sample by calculating a total immunostaining score as the product of a proportion and intensity score. The proportion score described the estimated fraction of positively stained tumor cells $(0=$ none; $1=<10 \%$; $2=10-50 \% ; 3=51-80 \% ; 4=>80 \%$ ). The intensity score represented the estimated staining intensity ( 0 , no staining; 1 , weak; 2 , moderate; 3 , strong). The total score ranged from 0 to 12 . Regarding the total score, the tissue samples were bimodally distributed with the nadir at a total score of 3-4. Therefore, TROP2 'overexpression' was arbitrarily defined as a total score $\geq 4$ as described previously. ${ }^{13}$ To assess the validity of the tissue microarray technique, we also analyzed full tissue sections using the same staining methods in a subset of 30 cases and matched them with the corresponding core sample. In $93 \%$ of the samples, analysis of a single punched core matched the staining pattern of a whole section.

\section{Statistical Methods}

Statistical analysis was performed using the Statistical Package of Social Science (SPSS, Version 10.0, 

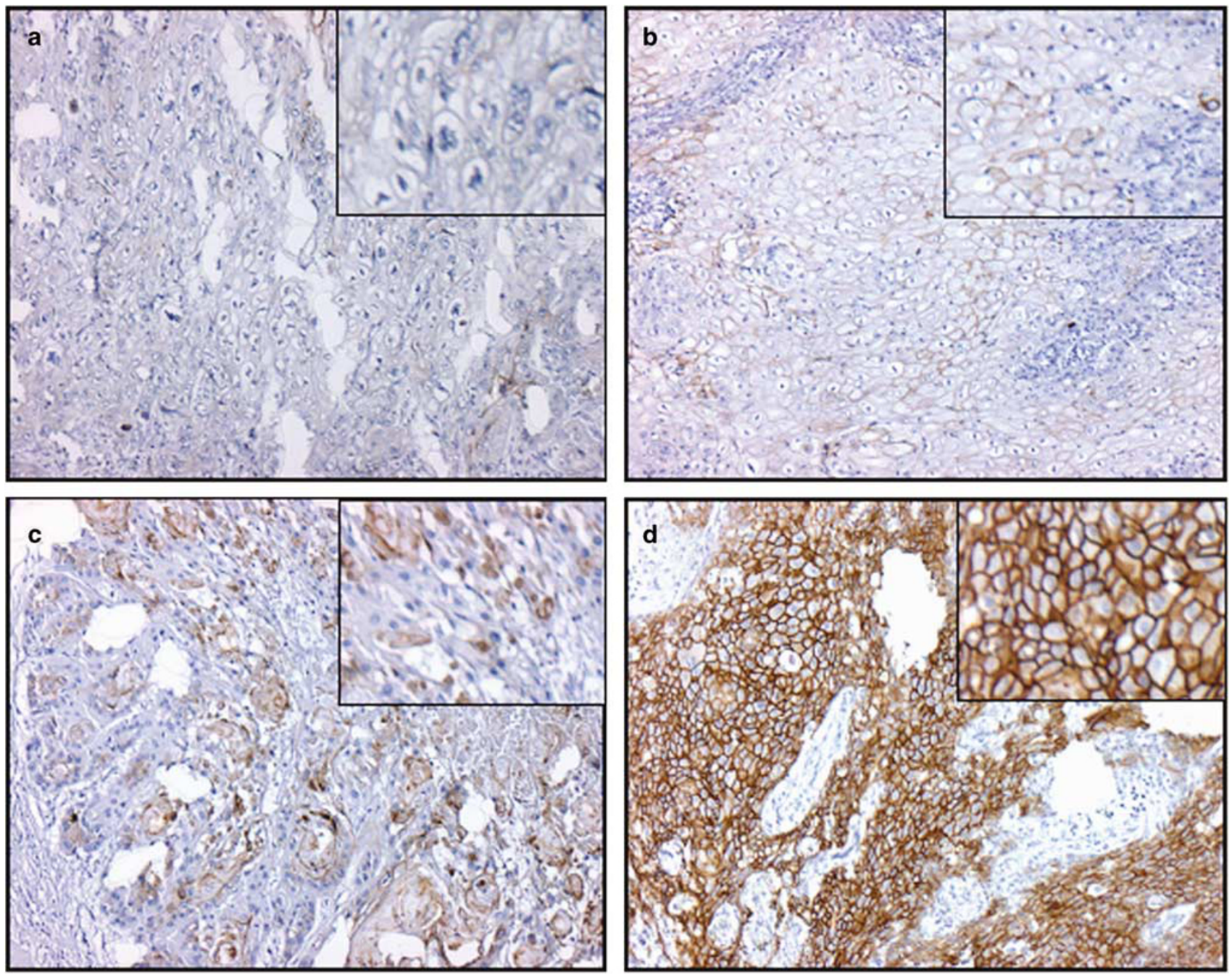

Figure 1 Immunohistochemical staining for TROP2 expression in oral squamous cell carcinoma; original magnification $\times 100$. Note the homogeneous membranous immunostaining of tumor cells. (a) TROP2-negative tumor sample (score 0); (b) weak expression (score 2); (c) moderate expression (score 6) and (d) strong expression (score 12).

Chicago, IL, USA). The primary end point in our study was overall survival. Survival curves were calculated using the method of Kaplan and Meier and compared by the log-rank test. Follow-up time was censored if the patient was lost to follow-up. The association between TROP2 expression and clinicopathological variables was assessed by the $\chi^{2}$-test. Factors shown to be of prognostic significance in the univariate models were evaluated in a multivariate Cox regression model. $P<0.05$ was considered statistically significant.

\section{Results}

\section{Patient Characteristics}

Demographic data and tumor characteristics are summarized in Table 1. A total of 90 patients were included in this retrospective study. Median overall survival was 23.8 months (range, 1-245). All patients underwent neck dissection and evalua- tion of lymph node status. The decision for adjuvant therapy was made according to individual considera tions, based on performance status, patient's compliance and clinicopathological features. R0-resection was accomplished in all patients.

\section{Immunohistochemistry}

As reported previously, TROP2 expression occurred primarily on the surface of neoplastic cells but not in adjacent normal epithelium. In contrast, a moderate to strong homogeneous membranous expression of TROP2 was noted in 52 (58\%) carcinoma specimens (Figure 1). By subgroup analysis, strong TROP2 expression (score 9-12) was found in 11 of $90(12 \%)$ cases, whereas moderate expression (score 6-8) occurred in 41 $(46 \%)$ cases and weak or no expression (score 0-4) in $38(42 \%)$ cases. Thus, according to the abovementioned criteria, TROP2 was found to be overexpressed in $52(58 \%)$ of 90 cases. 
Table 1 Patient characteristics

\begin{tabular}{|c|c|c|}
\hline \multirow[t]{2}{*}{ Variable } & \multicolumn{2}{|c|}{ Oral squamous cell carcinoma } \\
\hline & $(\mathrm{n}=90)$ & $(\%)$ \\
\hline \multicolumn{3}{|l|}{ Sex } \\
\hline Male & 71 & -79 \\
\hline Female & 19 & -21 \\
\hline \multicolumn{3}{|l|}{ Age } \\
\hline Mean & 63.4 & \\
\hline Median & 63.8 & \\
\hline Range & $25-85$ & \\
\hline \multicolumn{3}{|l|}{ Stage (UICC) } \\
\hline I & 6 & -7 \\
\hline II & 6 & -7 \\
\hline III & 21 & -23 \\
\hline IV & 57 & -63 \\
\hline \multicolumn{3}{|l|}{ Grade } \\
\hline 1 & 19 & -21 \\
\hline 2 & 49 & -54 \\
\hline 3 & 22 & -25 \\
\hline \multicolumn{3}{|l|}{ Median overall survival } \\
\hline Months & 23.8 & \\
\hline Range & $1-245$ & \\
\hline \multicolumn{3}{|l|}{ Tumor diameter } \\
\hline$<2 \mathrm{~cm}$ & 6 & -7 \\
\hline $2-4 \mathrm{~cm}$ & 23 & -26 \\
\hline$>4 \mathrm{~cm}$ & 29 & -32 \\
\hline Unknown & 32 & -35 \\
\hline \multicolumn{3}{|l|}{ Therapy } \\
\hline Surgery (R0-resection) & 90 & -100 \\
\hline Chemotherapy & 35 & -39 \\
\hline Radiotherapy & 27 & -30 \\
\hline \multicolumn{3}{|l|}{ TROP2 overexpression } \\
\hline Yes & 52 & -58 \\
\hline No & 38 & -42 \\
\hline
\end{tabular}

\section{Clinicopathologic Features and Patient Survival}

The association between TROP2 overexpression and clinicopathologic variables is shown in Table 2. By univariate analysis, comparing TROP2 overexpression to age, histologic grade, stage and lymph node metastasis, no correlation was found between TROP2 overexpression and conventional clinicopathologic features (Table 2). To assess the impact of clinicopathological features and TROP2 overexpression on survival, we used Kaplan-Meier analysis and the log-rank test for censored survival data. As expected, age, stage, histological grade and the presence of lymph node metastasis were of prognostic value. Of note, TROP2 overexpression was significantly associated with a poor overall survival $(P<0.01$; Figure 2$)$. Moreover, by subgroup analysis, overall survival gradually worsened with increasing TROP2 scores. The median survival decreased from 49 months in group 1 (score $0-4$ ) to 16 months in group 2 (score $5-8$ ), to 15 months in group 3 (score
Table 2 Correlation of TROP2 with conventional clinicopathological parameters

\begin{tabular}{|c|c|c|c|c|}
\hline \multirow[t]{2}{*}{ Characteristics } & \multirow[t]{2}{*}{ Patients } & \multicolumn{2}{|c|}{ TROP2 overexpression } & \\
\hline & & No & Yes & \\
\hline Grading & 90 & & & \\
\hline I & 19 & $10(53 \%)$ & $9(47 \%)$ & $P=0.46$ \\
\hline II & 49 & $18(37 \%)$ & $31(63 \%)$ & \\
\hline III & 22 & $10(45 \%)$ & $12(55 \%)$ & \\
\hline Clinical stage & 90 & & & \\
\hline I+II & 14 & $8(57 \%)$ & $6(43 \%)$ & $P=0.41$ \\
\hline $\mathrm{III}+\mathrm{IV}$ & 76 & $30(40 \%)$ & $46(60 \%)$ & \\
\hline Age & 90 & & & \\
\hline$<63$ & 40 & $15(37 \%)$ & $25(63 \%)$ & $P=0.41$ \\
\hline$>63$ & 50 & $23(46 \%)$ & $27(54 \%)$ & \\
\hline $\begin{array}{l}\text { Lymph node } \\
\text { metastasis }\end{array}$ & 90 & & & \\
\hline No & 42 & $19(45 \%)$ & $23(55 \%)$ & $P=0.60$ \\
\hline Yes & 36 & $13(36 \%)$ & $23(64 \%)$ & \\
\hline Unknown & 12 & $6(50 \%)$ & $6(50 \%)$ & \\
\hline
\end{tabular}

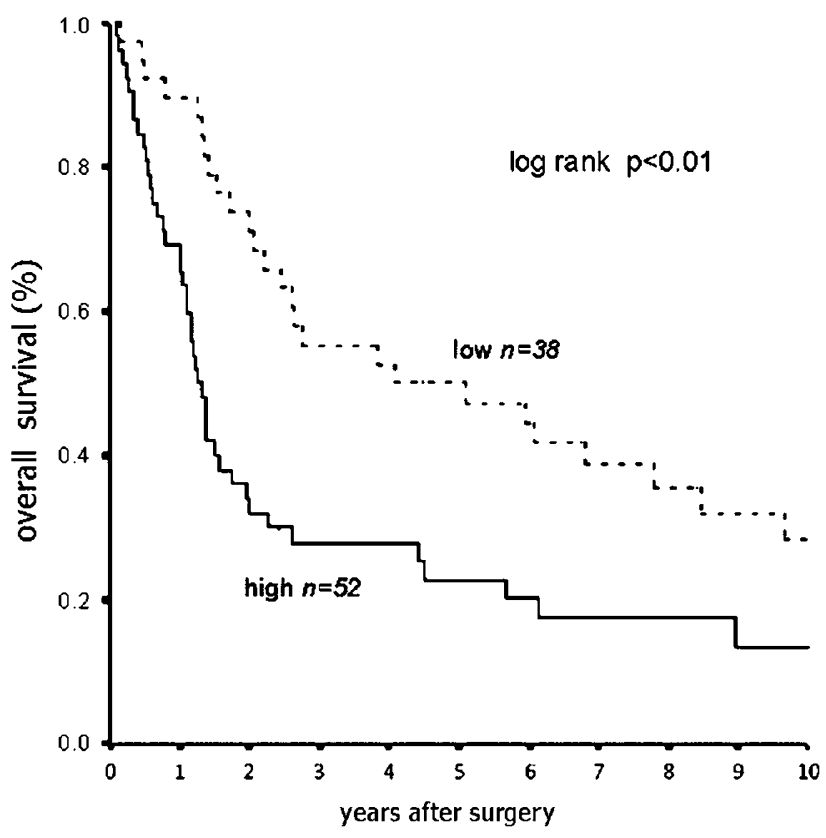

Figure 2 Prognostic significance of human trophoblast cellsurface antigen TROP2 overexpression in 90 patients with oral squamous cell carcinoma regarding overall survival by KaplanMeier analysis. High, patients with TROP2-overexpressing tumor $(n=52)$; low, patients with tumor $(n=38)$ without TROP2 overexpression. Patients with low TROP2 expression had a significantly better overall survival rate than patients with high TROP2 expression as defined by log-rank test $(P<0.01)$.

9-12) $(P<0.01$; Figure 3). Finally, all the statistically significant variables evaluated in the univariate analyses were included in a Cox proportional hazard analysis. Remarkably, TROP2 overexpression $(P<0.01$; RR, 2.26; 95\% CI, 1.33-3.83) along with patient's age, histologic grade and stage were identified to be an independent predictive factor for poor overall survival (Table 3 ). 
190

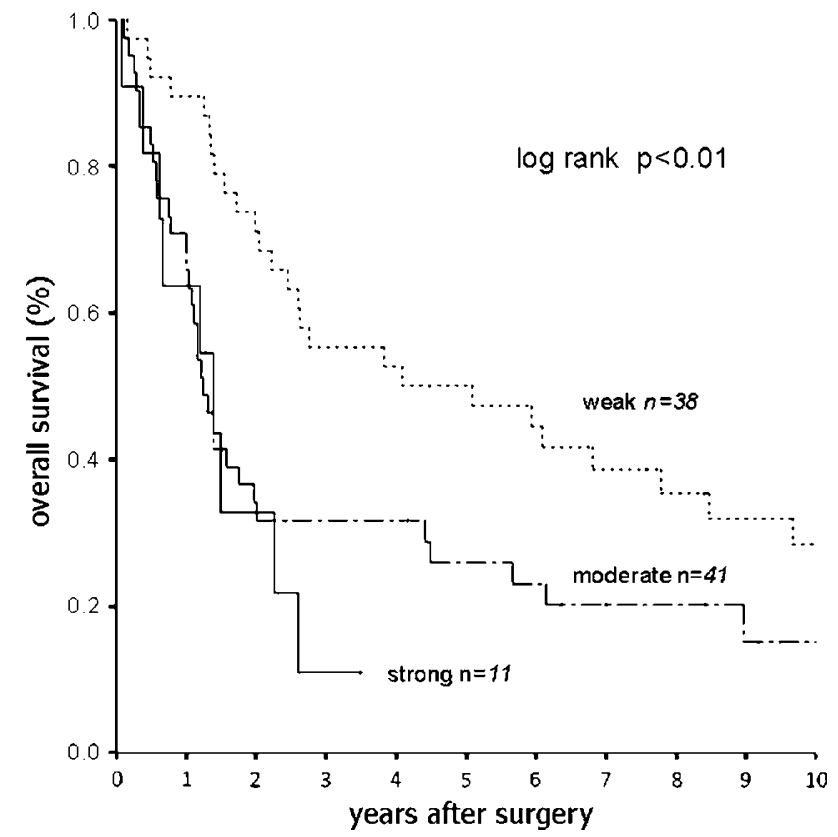

Figure 3 Prognostic significance of human trophoblast cellsurface antigen TROP2 expression in 90 patients with oral squamous cell carcinoma regarding overall survival by Kaplan-Meier analysis. By subgroup analysis, overall survival gradually worsened with increasing TROP2 scores. Patients lacking or with weak TROP2 expression $(n=38)$ had a significantly better overall survival than patients with moderate $(n=41)$ or strong $(n=11)$ TROP2 expression as defined by log-rank test $(P<0.01)$

Table 3 Multivariate analysis (Cox regression) of several prognostic markers in relation to survival of patients with oral squamous cell carcinoma

\begin{tabular}{lccc}
\hline & \multicolumn{3}{c}{ Overall survival } \\
\cline { 2 - 4 } & $\mathrm{P}$ & $R R$ & $95 \%$ CI \\
\hline Clinical stage (I, II vs III, IV) & 0.025 & 2.51 & $1.125-5.635$ \\
Grade & 0.045 & & \\
1 vs 2 & 0.015 & 0.36 & $0.168-0.826$ \\
1 vs 3 & 0.096 & 0.612 & $0.343-1.091$ \\
Lymph node metastasis & 0.074 & $\mathrm{NS}$ & \\
TROP2 overexpression & 0.002 & 2.26 & $1.338-3.839$ \\
Age at surgery & 0.001 & 2.52 & $1.482-4.315$ \\
(<63 vs >63 years) & & & \\
\hline
\end{tabular}

CI, confidence interval; NS, not significant; RR, relative risk.

\section{Discussion}

In this study, we first describe the prognostic relevance of TROP2 expression in patients with oral squamous cell carcinoma. Overexpression of TROP2 was detectable in 52 of 90 (58\%) specimens and significantly correlated with poor overall survival. In multivariate analysis, TROP2 overexpression was an independent prognostic marker.

The efficacy of classic chemotherapy with cisplatin/5 fluorouracil in patients with oral squamous cell carcinoma has reached a plateau. A better attention based on heterogeneity of the tumor and host characteristics might be one potential way to further improve outcome in these patients. Identification of new prognostic markers and target molecules may allow one to design patient-specific approaches for an optimal therapy. Targeting agents such as monoclonal antibodies have shown activity against different cancer types. This encourages the search for prognostic factors in order to select patients with an aggressive tumor phenotype for a specific treatment strategy.

TROP2, encoded by the single-exon gene TACSTD2, is highly homologous to the cell-cell adhesion molecule EpCAM (TACSTD1, GA733-2, 17-1A). The gene is expressed in various human carcinomas and its product is a type I transmembrane protein with several glycosylation sites. We recently showed that EpCAM (TROP1, GA733-2) overexpression predicts poor survival in different cancer entities. ${ }^{14-16}$ Interestingly, expression of TROP2 did not correlate with EpCAM expression. ${ }^{17}$ Anti-EpCAM antibodies can cause significant reduction in mortality and relapse rates of resected colon cancer patients following treatment with the EpCAM-specific monoclonal antibody edrecolomab. ${ }^{18}$ Owing to its immunogenic properties in cancer patients, active and passive immunotherapeutic agents against EpCAM antigen have been developed. ${ }^{19-21}$

The role of TROP2 is not well understood and the physiological ligand is still unknown. Although it has been previously suggested to function as a cell-cell adhesion receptor in cancer cells, recent data are rather indicative of a function in cell signal transduction and tumor cell growth. ${ }^{9,10}$ Terrinoni et $a l^{22}$ could isolate a novel cyclin D1-TROP2 fusion oncogene from human cancer cells, suggesting that TROP2 may play a role in the regulation of the cell cycle and the growth of carcinoma cells. Recently, TROP2 was found to be highly expressed in colorectal cancer correlating with aggressiveness and poor prognosis. ${ }^{23}$ Nakashima et $a l^{24}$ demonstrated that TROP2 is overexpressed in esophageal cancer and that serum TROP2 antibodies correlated with tumor size indicating that TROP2 could be a useful diagnostic marker. Furthermore, a murine monoclonal antibody (mAb) RS7, which specifically reacts with TROP2, has been developed and is rapidly internalized after binding to target cells. ${ }^{25} \mathrm{In}$ addition, a humanized version of RS7 (hRS7) was generated with promising results in an in vivo breast cancer model. ${ }^{26}$

Taken together, our data support the assumption that TROP2 plays an important role in tumor growth. However, due to the limitations inherent in retrospective analyses, the prognostic value of TROP2 overexpression needs to be validated in larger prospective studies. If confirmed in these studies, TROP2 may emerge as a prognostic tool and an attractive therapeutic target in a subgroup of patients with oral squamous cell carcinoma. 


\section{Acknowledgement}

We thank Ines Tschörner for excellent technical assistance. This work was supported by the ÖNB Grant 12168.

\section{Disclosure/conflict of interest}

There is no duality of interest to be disclosed.

\section{References}

1 Calabrese G, Crescenzi C, Morizio E, et al. Assignment of TACSTD1 (alias TROP1, M4S1) to human chromosome 2 p21 and refinement of mapping of TACSTD2 (alias TROP2, M1S1) to human chromosome 1p32 by in situ hybridization. Cytogenet Cell Genet 2001;92: 164-165.

2 Linnenbach AJ, Seng BA, Wu S, et al. Retroposition in a family of carcinoma-associated antigen genes. Mol Cell Biol 1993;13:1507-1515.

3 Linnenbach AJ, Wojcierowski J, Wu SA, et al. Sequence investigation of the major gastrointestinal tumor-associated antigen gene family, GA733. Proc Natl Acad Sci USA 1989;86:27-31.

4 Chong JM, Speicher DW. Determination of disulfide bond assignments and N-glycosylation sites of the human gastrointestinal carcinoma antigen GA733-2 (CO17-1A, EGP, KS1-4, KSA, and Ep-CAM). J Biol Chem 2001;276:5804-5813.

5 Lipinski M, Parks DR, Rouse RV, et al. Human trophoblast cell-surface antigens defined by monoclonal antibodies. Proc Natl Acad Sci USA 1981;78:5147-5150.

6 Alberti S, Miotti S, Stella M, et al. Biochemical characterization of Trop-2, a cell surface molecule expressed by human carcinomas: formal proof that the monoclonal antibodies $\mathrm{T} 16$ and $\mathrm{MOv}-16$ recognize Trop-2. Hybridoma 1992;11:539-545.

7 Stein R, Chen S, Sharkey RM, et al. Murine monoclonal antibodies raised against human non-small cell carcinoma of the lung: specificity and tumor targeting. Cancer Res 1990;50:1330-1336.

8 El Sewedy T, Fornaro M, Alberti S. Cloning of the murine TROP2 gene: conservation of a PIP2-binding sequence in the cytoplasmic domain of TROP-2. Int J Cancer 1998;75:324-330.

9 Ripani E, Sacchetti A, Corda D, et al. Human Trop-2 is a tumor-associated calcium signal transducer. Int J Cancer 1998;76:671-676.

10 Fornaro M, Dell'Arciprete R, Stella M, et al. Cloning of the gene encoding Trop-2, a cell-surface glycoprotein expressed by human carcinomas. Int J Cancer 1995;62: 610-618.

11 Sozo F, Wallace MJ, Zahra VA, et al. Gene expression profiling during increased fetal lung expansion identifies genes likely to regulate development of the distal airways. Physiol Genomics 2006;24:105-113.

12 Shimada A, Kano J, Ishiyama T, et al. Establishment of an immortalized cell line from a precancerous lesion of lung adenocarcinoma, and genes highly expressed in the early stages of lung adenocarcinoma development. Cancer Sci 2005;96:668-675.

13 Gastl G, Spizzo G, Obrist P, et al. Ep-CAM overexpression in breast cancer as a predictor of survival. Lancet 2000;356:1981-1982.

14 Spizzo G, Obrist P, Ensinger C, et al. Prognostic significance of Ep-CAM AND Her-2/neu overexpression in invasive breast cancer. Int J Cancer 2002;98: 883-888.

15 Fong D, Steurer M, Obrist P, et al. Ep-CAM expression in pancreatic and ampullary carcinomas: frequency and prognostic relevance. J Clin Pathol 2006, (e-pub ahead of print).

16 Varga M, Obrist P, Schneeberger S, et al. Overexpression of epithelial cell adhesion molecule antigen in gallbladder carcinoma is an independent marker for poor survival. Clin Cancer Res 2004;10:3131-3136.

17 Laimer K, Fong D, Gastl G, et al. EpCAM expression in squamous cell carcinoma of the oral cavity: frequency and relationship to clinicopathologic features. Oral Oncol 2007 (e-pub ahead of print).

18 Riethmuller G, Holz E, Schlimok G, et al. Monoclonal antibody therapy for resected Dukes' C colorectal cancer: seven-year outcome of a multicenter randomized trial. J Clin Oncol 1998;16:1788-1794.

19 Mosolits S, Markovic K, Frodin JE, et al. Vaccination with Ep-CAM protein or anti-idiotypic antibody induces Th1-biased response against MHC class Iand II-restricted Ep-CAM epitopes in colorectal carcinoma patients. Clin Cancer Res 2004;10:5391-5402.

20 de Bono JS, Tolcher AW, Forero A, et al. ING-1, a monoclonal antibody targeting Ep-CAM in patients with advanced adenocarcinomas. Clin Cancer Res 2004;10:7555-7565.

21 Samonigg H, Himmler M, Gnant M. A multicenter phase II, open-label trial of multiple doses of cancer vaccine candidate IGN101 to evaluate efficacy against disseminated tumor cells in blood [abstract]. J Clin Oncol 2005;23:180S

22 Terrinoni A, Dell'Arciprete R, Fornaro M, et al. Cyclin D1 gene contains a cryptic promoter that is functional in human cancer cells. Genes Chromosomes Cancer 2001;31:209-220.

23 Ohmachi T, Tanaka F, Mimori K, et al. Clinical significance of TROP2 expression in colorectal cancer. Clin Cancer Res 2006;12:3057-3063.

24 Nakashima K, Shimada H, Ochiai T, et al. Serological identification of TROP2 by recombinant cDNA expression cloning using sera of patients with esophageal squamous cell carcinoma. Int J Cancer 2004;112: 1029-1035.

25 Stein R, Basu A, Chen S, et al. Specificity and properties of MAb RS7-3G11 and the antigen defined by this pancarcinoma monoclonal antibody. Int J Cancer 1993;55:938-946.

26 Govindan SV, Stein R, Qu Z, et al. Preclinical therapy of breast cancer with a radioiodinated humanized antiEGP-1 monoclonal antibody: advantage of a residualizing iodine radiolabel. Breast Cancer Res Treat 2004;84: 173-182. 